\title{
Impacto en el proceso de selección de los nuevos médicos residentes de neonatología por la pandemia por COVID-19
}

\author{
Impact on the selection process of new neonatology residents due to the \\ COVID-19 pandemic
}

La pandemia por SARS-COV-2 que nos encontramos viviendo ha modificado radicalmente la forma en la que se venía desarrollando la medicina y por ende las actividades cotidianas. Se pasó de tener un contacto estrecho y cercano persona-persona a una protección extrema e incómoda, pero necesaria. Diversos son los rubros de la sociedad que se han visto modificados y uno de ellos ha sido la convivencia académica médica en donde las sesiones, entregas de guardia, congresos y seminarios no han permanecido de la manera en la que las conocíamos.

La educación médica se ha tenido que adaptar debido a la alta demanda asistencial que exige esta contingencia, y a la necesidad de continuar con un programa académico. Esto ha permitido, al proceso de enseñanza, convertirse en un agente activo y dinámico, predominantemente virtual y no así a la actividad asistencial. ${ }^{1}$

La preparación de los nuevos médicos y especialistas no ha quedado exenta de esta nueva normalidad; jóvenes médicos que tendrían que estar aprendiendo a tratar diversas enfermedades han tenido que dedicar el $100 \%$ de su tiempo únicamente al cuidado de pacientes contagiados por SARS-COV-2 y sus complicaciones.

Como es evidente los hospitales participan en el proceso de formación de diversos médicos en las diferentes ramas de la medicina, entre las cuales, a pesar de la pandemia, la sociedad continúa requiriendo el apoyo de servicios que no son directa o indirectamente afectados por la pandemia. Esto ha requerido su continuidad operativa y en algunas situaciones con sobrecarga de trabajo debido al cierre de algunos otros centros para ser convertidos en Hospitales COVID. ${ }^{2}$

Todos estos médicos pasan por un riguroso proceso de selección para ser admitidos a cada uno de los programas de especialidades médicas en donde aprueban exámenes de conocimientos médicos, conocimientos generales, pruebas psicométricas y entrevistas con los claustros de profesores de la especialidad que han decidido cursar, y que se han tenido que adaptar para salvaguardar la salud y el bienestar de todos los involucrados en dichas actividades.

La entrevista presencial a candidatos ha sido un instrumento para la identificación de los aspirantes a especialidades médicas; la personalidad, el comportamiento y su actitud ante ciertos cuestionamientos han formado parte del proceso de selección. Durante la pandemia hemos tenido que migrar a entrevistas virtuales en donde se pierde esa interacción persona a persona y en donde se permite al entrevistado reaccionar de manera diferente frente a la cámara de su computadora o tableta, lo que ocasiona una disminución de la tensión visual e inquisidora de algunos entrevistadores y por ende se pierde algún detalle del entrevistado.

Sin embargo, no todo es negativo; con las entrevistas en línea, se incrementa la posibilidad de aspirantes de diversas partes del mundo ante la facilidad que da la conexión virtual, así como de programar a dichos entrevistados un mismo día y evitar su traslado. En el grupo de entrevistadores también mejora la presencia de múltiples entrevistadores localizados también en diferentes puntos.

Sólo con el tiempo podremos evidenciar las consecuencias de las nuevas opciones digitales a las que ha migrado la educación médica y su impacto o no en la práctica clínica y la salud de sus comunidades; algunas especialidades médicas han tenido que suspender las actividades propias por atender a los pacientes afectados por la pandemia por SARS-COV-2.

Dr. Bronson Osorio Martínez $z^{a}$ Dr. Jorge A. Martínez Cardona ${ }^{b}$

a. Pediatra y Neonatólogo, Director del Programa de Neonatología, Programas Multicéntricos de Especialidades Médicas, Tecnológico de Monterrey. Monterrey, Nuevo León, México.

b Pediatra, Residente de Neonatología, Programas Multicéntricos de Especialidades Médicas, Tecnológico de Monterrey. Monterrey, Nuevo León, México.

Correspondencia:

Dr. Jorge A. Martínez Cardona: jorge.mtzcardona@gmail.com

http:/ / dx.doi.org/10.5546/aap.2021.150

Texto completo en inglés:

http: / / dx.doi.org/10.5546/aap.2021.eng.150 
Cómo citar: Osorio Martínez B, Martínez Cardona JA. Impacto en el proceso de selección de los nuevos médicos residentes de neonatología por la pandemia por COVID-19. Arch Argent Pediatr 2021;119(3):150-151

\section{REFERENCIAS}

1. Rose S. MedicalStudent Education in the Time of COVID-19. JAMA. 2020;323(21):2131-2.

2. Ayanian JZ, Weissman JS. Teaching hospitals and quality of care: a review of the literature. Milbank Q. 2002;80(3):569-93, v.

\section{Archivos hace 75 años}

PERICARDITIS PURULENTA TRATADA CON PENICILINA. OBSERVACION DE UNA NINA DE DOS MESES DE EDAD*

rox 105

Dass, J. P. GARRAHAN, G. F. THOMAS y J. A. CAPRILE

El 30 de abril de 1945 ingresa al Servicio de Lactantes del Instituto en que actuamos, una niña de dos meses de edad (Historia $\mathrm{N}$ ? 21.752), nàcida a término con $4.100 \mathrm{~g}$. de peso y criada por su madre. Sin antecedentes de importancia, una semana antes se había enfermado con fiebre y catarro de vias aéreas superiores, que en los últimos dias habiase agravado, motivo por el cual se la traía para internar. Fué tratada primero con sulfadiazina a dosis insuficientes. Al agravarse, se le suministró sulfamerazina $0,12 \mathrm{~g}$. cada 5 horas. Con esta terapécutica la fiebre desapareció, pero la niña se mantuvo muy postrada, disneica y cianótica. El médico que la trataba encontró respiración brónquica en el vértice pulmonar derecho.

Al ser examinada se comprueba lo siguiente:

Adelgazamiento, disnea, cianosis peribucal, mirada indiferente y vaga.

Submatitez en la mitad inferior de la cara posterior del hemitórax izquierdo. En la misma zona se ausculta respiración brónquica con broncofonia.

Hay taquicardia. El latido de la punta no se ve ni se palpa. La matidez absoluta está aumentada. Los ruidos cardiacos se perciben como alejados.

EI abdomen excursiona con los movimientos respiratorios. Es globuloso y depresible. El hígado se palpa indoloro, a nivel de la cresta iliaca.

La telerradiografia muestra una sombra cardiaca enormemente agrandada y globuloxa - "en botellón" - con ángulo cardiohepático agudo y éstasis pulmonar. La relación cardiotorícica era de 76 \% (Fig. 1).

Se diagnostica derrame pericárdico. El electrocardiograma revela una sobreelevación del segmento ST en la primera y segunda derivación, con ondas $\mathbf{T}$ aplanadas (Fig. 2).

La punción del pericardio, realizada por vía epigástrica, permite extraer 30 c.c. de liquido purulento, con abundante cantidad de estafilococos y escasos estreptococos de cadena corta. Se inyectan en la cavidad cardiaca, $1,7 \mathrm{gr}$. de cibazol soluble y se administran 10.000 unidades de penicilina, por vía intramuscular, cada 4 horas. La niña succiona mejor y la disnea y la cianosis son menos intensas. La telerradiografia efectuada inmediatamente después no muestra diferencias con la anterior.

Al dia siguiente el hígado se mantiene a tres traveses de dedo del reborde costal. Se decide hacer una nueva punción; esta vez se sacan 75 c.c. de pus achocolatado y grumoso, necesitándose inyectar para ello 20 c.c. de suero fisiológico; para evitar adherencias se inyectan 20 c.C. de aire, en la cavidad pericárdica. La telerradiografia evidencia el saco pericárdico distendido, por el

$$
\text { - Com }
$$

- Comunicación presentada a la Sociedad Argentina de Pediatria en la sesión del 14 de agosto de 1945. 